\title{
Structure Optimization of Coded Polyphase Signals with the Enhanced Tolerance to the Doppler Frequency Shift
}

\author{
D. SzCZEGIELNIAK ${ }^{a, *}$, A. MILEWSKi ${ }^{b}$ AND M. SzCZEGIELNIAK ${ }^{a}$ \\ ${ }^{a}$ Faculty of Telecommunications, Computer Science and Electrical Engineering, \\ University of Technology and Life Sciences, al. S. Kaliskiego 7, 85-789 Bydgoszcz, Poland \\ ${ }^{b}$ Tele \& Radio Research Institute, Ratuszowa 11, 03-450 Warszawa, Poland \\ (Received February 24, 2015)

\begin{abstract}
The aim of this research was to verify if It is possible to solve the synthesis problem of polyphase codes efficiently with an acceptable quality in the context of radar applications - with the greatest possible effectiveness of the recognition of such signals in the presence of the noise and occurrence of the Doppler effect. The received results confirmed that the implemented optimization algorithm can search polyphase sequences successfully with a low level of sidelobes and an enhanced tolerance to the Doppler effect. Applying optimization methods allows to form ambiguity function in a measure in the assumed optimization range which seems to be encouraging for future examinations in the field of the computer optimization for the coded radar signals synthesis.
\end{abstract}

DOI: 10.12693 /APhysPolA.128.452

PACS: $84.40 . \mathrm{Ua}, 84.40 . \mathrm{Xb}$

\section{Introduction}

The search of polyphase sequences with the enhanced tolerance to a Doppler frequency offset in the received signal is computationally complex and based on the analysis of the ambiguity function. This two-dimensional function presents the influence of the Doppler effect on the correlation result executed in radar receivers by means of the matched filter

$$
\left|\chi\left(\tau, f_{d}\right)\right|^{2}=\left|\int_{-\infty}^{\infty} u(t) u^{*}(t-\tau) \mathrm{e}^{\mathrm{j} 2 \pi f_{d} t} \mathrm{~d} t\right|^{2} .
$$

Inserting the equation of the polyphase signal envelope

$$
u(t)=\frac{1}{\sqrt{T}} \sum_{m=1}^{M} \mathrm{e}^{\mathrm{j} \phi_{m}} \operatorname{rect}\left(\frac{t-(m-1) t_{b}}{t_{b}}\right)
$$

to the formula which defines the ambiguity function, we get

$$
\chi\left(\tau, f_{d}\right)=
$$$$
\frac{1}{M} \sum_{n=0}^{M-1} \sum_{m=0}^{M-1} e^{j 2 \pi f_{d} n t_{b}} e^{j\left(\phi_{n}-\phi_{m}\right)} \chi_{b}\left(\tau-(n-m) t_{b}, f_{d}\right),
$$

where $\chi_{b}\left(\tau ; f_{d}\right)$ is the ambiguity function of every single code element - the triangular function along the axis of the delay $\tau$ in the interval $\pm t_{b}$ and the sinc function on the frequency axis $f_{d}[1-3]$.

The ambiguity function is commonly used by radar systems designers as a tool for the research and analysis of different types of radar signals, allowing to specify the range resolution of particular radar signals as well as the Doppler resolution (connected with the Doppler effect caused by the speed of the moving object).

\footnotetext{
*corresponding author; e-mail: dszczeg@utp.edu.pl
}

Moreover, the ambiguity function provides the information of in what type of radar applications a given radar signal could be used. Unfortunately, there are no analytical methods of the radar signals synthesis based on the ambiguity function. Therefore, designing a signal with some desirable properties of the ambiguity function is based mainly on the knowledge and experience of the radar systems designer.

The function has relevant and important property caused by so-called linear frequency modulation effect, which corresponds to the quadratic phase modulation in the polyphase signals. This property relies on the fact that the signal compression is still present even in an area for divergent frequencies between the stored replica of the sent signal and received reflected signal affected by the Doppler effect. In this case the mainlobe on the chart of the ambiguity forms a ridge (see Fig. 1). In many applications where the Doppler shift is relatively small the mistake connected with the range resolution is acceptable and can be ignored. Therefore, this kind of the ambiguity function is desirable in such applications because of the enhanced tolerance to the Doppler effect.

In the case of radar signals where the mainlobe of the ambiguity function forms a ridge, reaching high values of the Doppler frequencies (for example, linear frequency modulated signal or polyphase P-codes), calculated distance on the basis of echoed signal from a moving object affected by the Doppler effect can be vitiated by an unacceptable error in practice. These kind of signals, depending on their applications and a situation, do not have to be considered as ones with the enhanced tolerance to the Doppler effect. However, in many radar applications these kinds of the radar signals are profitable.

The tolerance to the Doppler effect is especially important when the long-duration pulses are sent in order to detect objects at high speeds. If in the received radar signal a frequency shift appears we can expect a much 


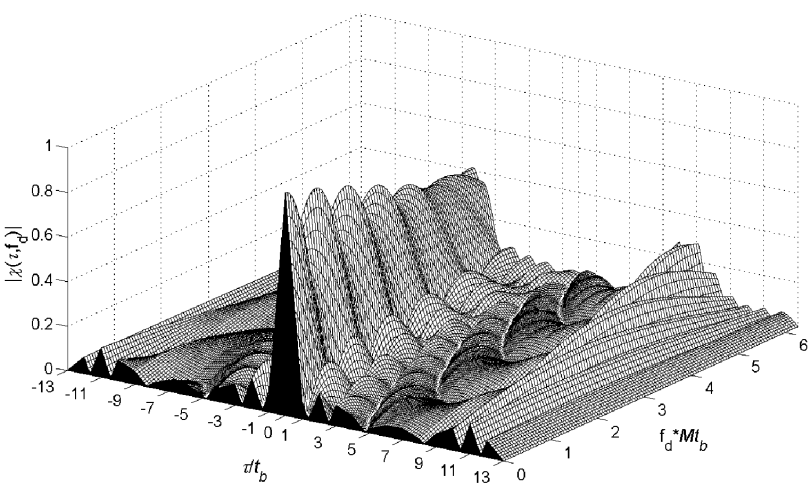

Fig. 1. The ambiguity function of the thirteen-element $\mathrm{P} 4$ code, $\mathrm{PSL}=-17.31 \mathrm{~dB}$ for $f_{d}=0$.

higher level of sidelobes than estimated earlier by means of the autocorrelation function.

Generally, polyphase codes are sensitive to the Doppler shift, whereas the enhanced tolerance of P4 codes (including the Frank code as well as other P-codes) results from the fact that these sequences were created by the sampling of the phases of signals with the linear (or stepped) frequency modulation which is known for their tolerance to the Doppler shift $[4,5]$.

The aim of our research was to verify if It is possible to solve the synthesis problem of the polyphase codes efficiently with an acceptable quality in the context of radar applications - with the greatest possible effectiveness of the recognition of such signals in the presence of the noise and occurrence of the Doppler effect.

\section{Optimization method}

Optimization tasks addressed in this paper were nonlinear NP-hard optimization problems with continuous variables and many local optima [6]. For these timeconsuming and computationally complex problems applying methods of the full search of the space of possible solutions (where their number increases exponentially with the length of the sought code) is unacceptable in practice due to the enormous execution time and hardware requirements related to the execution of such tasks.

Taking advantage of heuristic optimization methods is a lot more effective and It seems to be a reasonable compromise between the quality of the found solution and required cost of executing the task.

However, It is very important here to choose appropriate methods to solve the problem. Applying an improper method can result in lack of the convergence of the optimization process and may resemble a fully random search of the desirable solution without taking any advantage of the efficiency of heuristic or even traditional optimization methods.

To solve the optimization problem of the polyphase signals structure, the evolutionary algorithm based on a floating-point representation was implemented. Each individual in the population was represented as a vector of floating-point numbers $\boldsymbol{x}=\left(x_{1}, x_{2}, \ldots, x_{n}\right)$. For such a complicated nonlinear optimization problem which is the synthesis of the coded signal structure, the crossover operator was rejected because of its disruptive influence on the convergence of the algorithm. In order to produce offspring for the next generation the Gaussian mutation was used.

Mutations were then realized by adding to each component of the vector a random Gaussian number with the zero mean value and standard deviation $\sigma_{i}$, changing during the evolutionary process and controlled by the self-adaptation mechanism. In this scheme, each individual has its own adaptable vector of $\sigma=\left(\sigma_{1}, \sigma_{2}, \ldots, \sigma_{n}\right)$ values, which learns itself how to search the space of potential solutions. The applied self-adaptation mechanism allows for a greater freedom, adapting the search strategy to the topology of the fitness landscape. The mutations were realized according to formula

$$
\begin{aligned}
\sigma_{i}^{\prime} & =\sigma_{i} \mathrm{e}^{N(0, \tau)}, \\
x_{i}^{\prime} & =x_{i}+N\left(0, \sigma_{i}^{\prime}\right),
\end{aligned}
$$

where $\tau$ is a parameter of the method that was set to $1 / \sqrt{n}$ and $n$ is the size of the problem [7].

For the selection of individuals for the next generation, the tournament selection was applied. In this approach, the individuals in the population are randomly grouped in pairs and then the fitness levels of two individuals are compared with each other. The individual with the better fitness survives to the next iteration while this second one is discarded.

\section{Results and discussion}

An example of ambiguity function of the thirteenelement polyphase sequence obtained in the optimization process which takes into account the tolerance to the Doppler effect is depicted in Fig. 2. The evolutionary algorithm described in Sect. 2 was seeking (with the defined step in the selected Doppler frequency ranges) the correlation function with the smallest ratio of the sidelobes level to the mainlobe level.

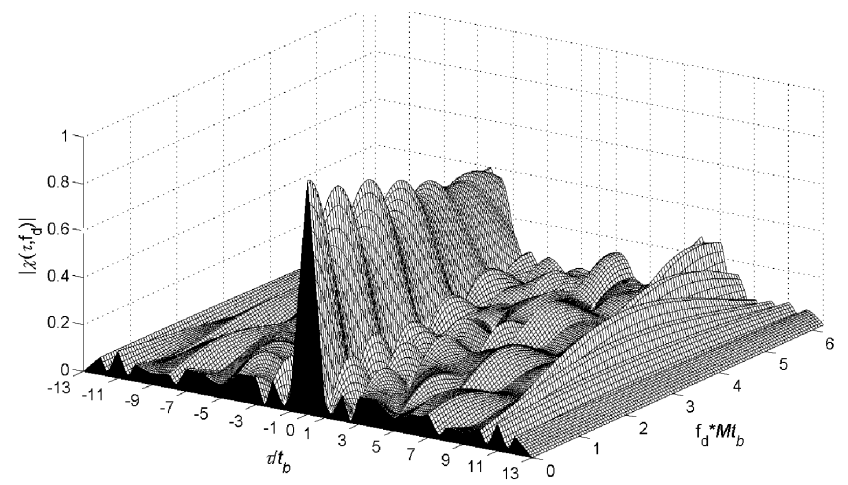

Fig. 2. The ambiguity function of the thirteen-element polyphase sequence obtained in the optimization process, taking into account the Doppler effect, PSL = $-18.55 \mathrm{~dB}$ for $f_{d}=0$. The result of the optimization within the range $f_{d} M t_{b} \in\langle 0 ; 2.5\rangle$. 


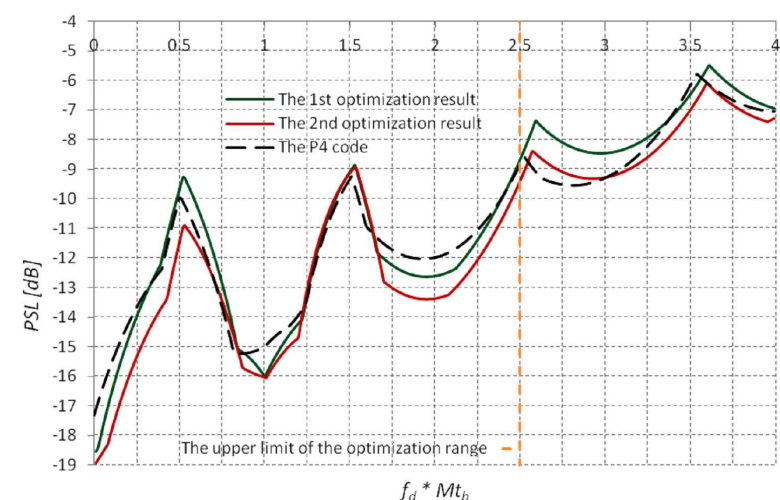

Fig. 3. The ratio of sidelobes levels to ridge lines of ambiguity functions of thirteen-element polyphase sequences. Optimization results within the range $f_{d} M t_{b} \in$ $\langle 0 ; 2.5\rangle$.

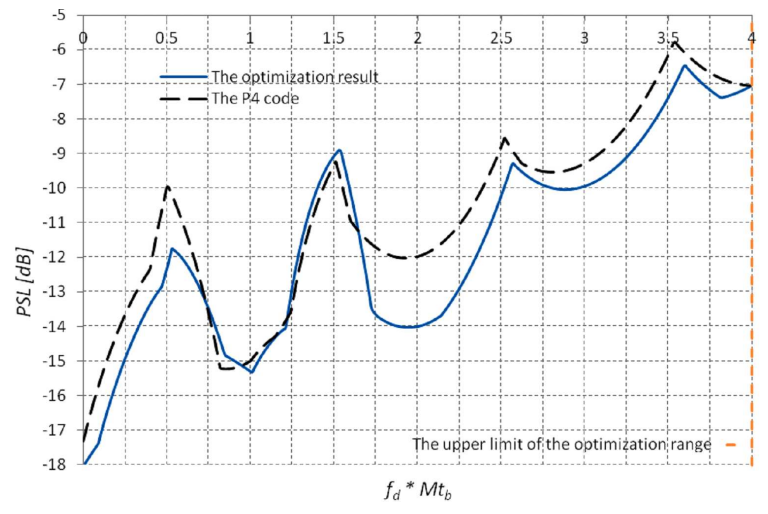

Fig. 4. The ratio of sidelobes levels to ridge lines of ambiguity functions of thirteen-element polyphase sequences. Optimization results within the range $f_{d} M t_{b} \in$ $\langle 0 ; 4\rangle$.

In Figs. 3 and 4 three different thirteen-element polyphase sequences, being results of the optimization, were compared with the P4. For zero Doppler frequencies all obtained codes are slightly better than the P 4 code. Differences were from $0.64 \mathrm{~dB}$ for sequences in the widest frequency range to $1.63 \mathrm{~dB}$ for another optimization result. Generally, obtained codes in the whole optimization ranges were a little bit better or comparable with the $\mathrm{P} 4$ code. However, It is worth mentioning that there was no case where optimization results were much worse. Obtained results confirmed that the implemented optimization algorithm could search for polyphase sequences efficiently with low sidelobes levels and enhanced tolerance to the Doppler effect.

As it can be noted in Fig. 2 and 5 the compression effect of the pulse remains outside the Doppler frequency range, for which the optimization was carried out. This is due to the linear frequency modulation effect which corresponds to the quadratic phase modulation in sequences obtained in the optimization process for a quite limited range of the Doppler frequencies. Each of found codes had phase

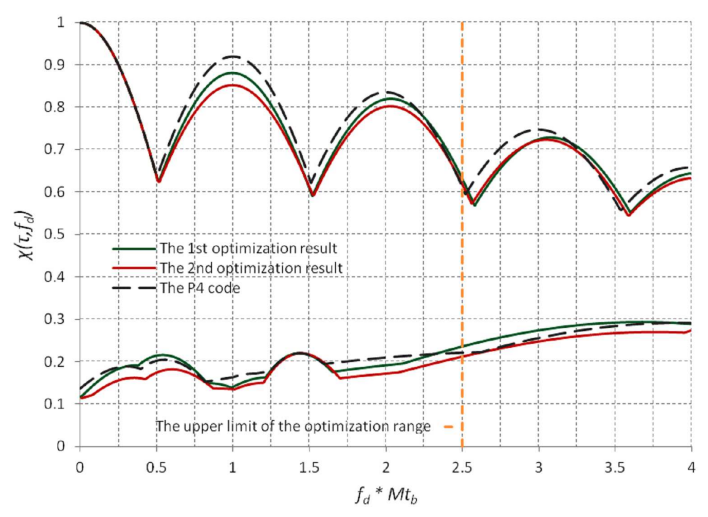

Fig. 5. Levels of ridge lines and sidelobes of ambiguity functions of 3 different thirteen-element polyphase sequences. Optimization results within the range $f_{d} M t_{b} \in$ $\langle 0 ; 2.5\rangle$.

sequences with the distribution similar to the quadratic phase modulation (in order to note this It is necessary to add $\pm 360^{\circ}$ to proper elements of the code). A similar relationship exists in the case of the phases distribution of polyphase codes obtained from the phase sampling of the signal with the frequency modulation and known for their enhanced tolerance to the Doppler effect (referred to in the literature as chirp-like codes such as $\mathrm{P} 1, \mathrm{P} 2 / \mathrm{Px}$, P3, P4).

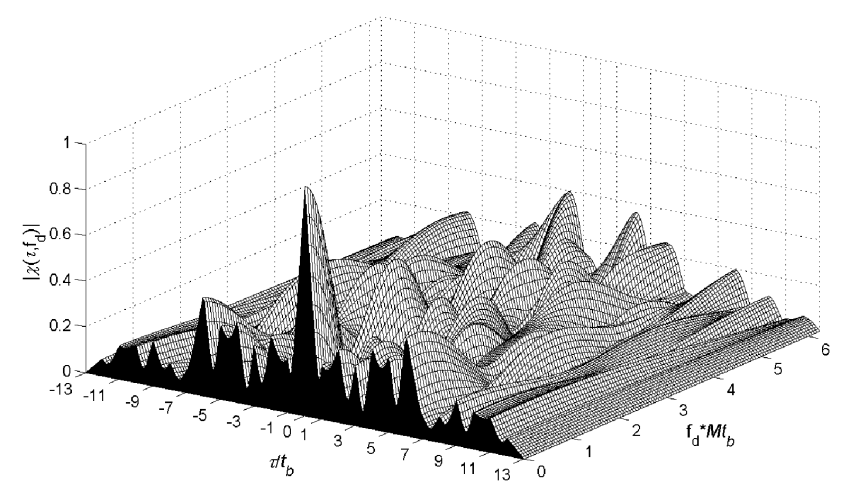

Fig. 6. The ambiguity function of thirteen-element polyphase sequences generated randomly, being an example of a possible individual for the initial generation (not processed by the evolutionary algorithm) with PSL $=-8.13 \mathrm{~dB}$ for $f_{d}=0$.

The optimization process did not affect the phase distribution directly. The objective function of the optimization algorithm is based on forming the ambiguity function in a particular way within a specified Doppler frequency range. In this case it could be noted that initial phase sequences generated randomly (the ambiguity function of a sample initial sequence is shown in Fig. 6) during the optimization process were taking the form of the approximated distribution of the quadratic phase modulation, adjusting themselves to the specified shape of the ambiguity function simultaneously. 
For each code length the optimization can be performed in two ways i.e., for the ridge line passing toward positive or negative delay values of $\tau$. It results in the characteristic distribution of phases, resembling a phases sampling of the type of chirp-up signals with the increasing frequency or chirp-down with the decreasing frequency linearly. In Fig. 7 the first result of the optimization and the P4 code have phase sequences that resemble phase samples of the chirp-down signal and chirpup one in the case of the rest of results.

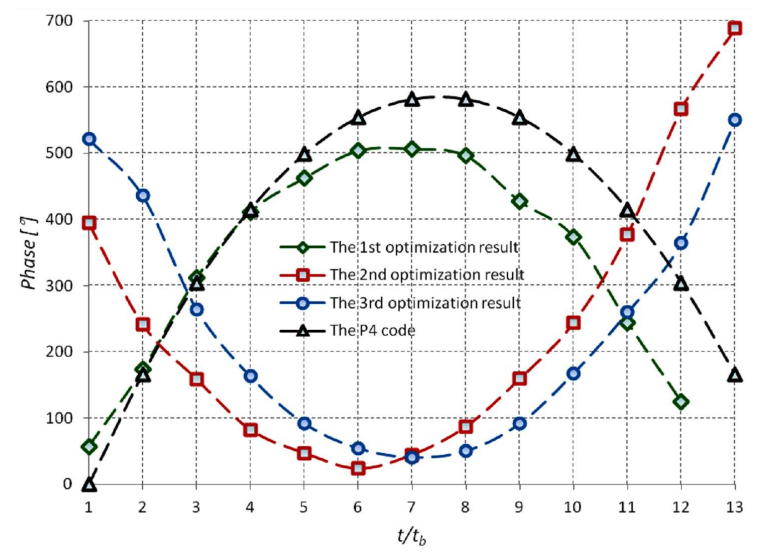

Fig. 7. Thirteen-element polyphase sequences obtained in the optimization process and the $\mathrm{P} 4$ code. In the case of some optimization resultant elements $\pm 360^{\circ}$ values were added in order to illustrate the relationship of phase signal samples with the linear frequency modulation.

\section{Conclusions and remarks}

The carried out research confirms that applying optimization methods can resolve effectively the search of polyphase sequences with desirable autocorrelation function properties as well as with the enhanced resistance to the Doppler effect. The results seem to be very encouraging for future examinations in the field of the computer optimization for the synthesis of coded radar signals. For sure, the challenge is to search very long codes because of the computational complexity and time consumption (a number of possible solutions increase exponentially with the length of the code). Applying optimization methods for searching sequences with the enhanced tolerance to the Doppler effect allows to form the ambiguity function in a measure in an assumed optimization range.

\section{References}

[1] B.R. Mahafza, A.Z. Elsherbeni, in: MATLAB Simulations for Radar Systems Design, Chapman and Hall/CRC, 2003, p. 203.

[2] N. Levanon, E. Mozeson, in: Radar Signals, Wiley, 2004, p. 34; 100.

[3] T. Misaridis, Ultrasound Imaging Using Coded Signals, Technical University of Denmark, 2001, p. 61.

[4] B.L. Lewis, F.F. Kretschmer, IEEE Trans. Aerospace Electron. Syst. AES-18, 637 (1982).

[5] T. Felhauer, IEEE Trans. Aerospace Electron. Syst. 30, 869 (1994).

[6] N. Mladenovic, J. Petrovic, V. Kovacevic-Vujcic, M. Cangalovic, Europ. J. Operat. Res. 151, 389 (2003).

[7] Z. Michalewicz, D.B. Fogel, in: How to Solve It: Modern Heuristics, Springer Verlag, 2000, p. 276. 